\title{
AVALIAÇÃO DO PROCESSO FÍSICO DE CONGELAMENTO DA POLPA DE LIMÃO TAHITI (Citrus latifólia Tanaka)
}

\author{
K. D. Brito ${ }^{*}$, L. S. C. Oliveira ${ }^{2}$, A. G. B. de Lima ${ }^{3}$,A. M. Santiago ${ }^{4}$, D. E. G. Alves ${ }^{5}$ \\ 1 Instituto Federal de Educação, Ciência e Tecnologia - IFET/PB - Campus Campina Grande \\ ${ }^{2}$ Universidade Federal de Campina Grande - PB, Departamento de Engenharia Química \\ ${ }^{3}$ Universidade Federal de Campina Grande - PB, Departamento de Engenharia Mecânica \\ ${ }^{4}$ Universidade Estadual da Paraíba, Departamento de Química \\ ${ }^{5}$ Graduando em Engenharia de Alimentos, Universidade Federal de Campina Grande -PB \\ *E-mail para contato: katiadout@ hotmail.com
}

\begin{abstract}
RESUMO - O processo de congelamento é uma prática muito utilizada na obtenção de polpas de frutas, consistindo em mais uma alternativa para a utilização de frutas que são comercializadas na maioria das vezes na forma in natura, cujo preço não é compensador. O limão Tahiti (Citrus latifolia Tanaka), que tem sua produção nacional destinada prioritariamente à comercialização do produto in natura, vem crescendo em importância no grupo dos citros, entretanto seu processamento em suco concentrado congelado ainda é pequeno. O presente trabalho tem como objetivo o estudo do processo físico de congelamento da polpa de limão Tahiti, para a determinação do tempo e temperatura de congelamento e estruturação dos cristais de gelo. A polpa de limão Tahiti foi congelada a $-20 \pm 2{ }^{\circ} \mathrm{C}$ em um freezer doméstico. As temperaturas de resfriamento, congelamento e pós-congelamento foram coletadas utilizando um sistema de aquisição de dados, para o estudo da cinética de congelamento. A microestrutura dos cristais de gelo foi observada após o congelamento da polpa por meio da microscopia óptica. Os resultados mostraram que as temperaturas de início de congelamento foram de $-1,024^{\circ} \mathrm{C},-1,045^{\circ} \mathrm{C} \mathrm{e}-1,032^{\circ} \mathrm{C}$ correspondendo aos tempos de 1999, 2280 e 2000 segundos, respectivamente. As microestruturas dos cristais de gelo apresentaram morfologias de uma rede de dendritas crescendo paralelamente.
\end{abstract}

\section{INTRODUÇÃO}

O limão Tahiti (Citrus latifolia Tanaka), que tem sua produção nacional destinada prioritariamente à comercialização do produto in natura, vem crescendo em importância no grupo dos citros, entretanto seu processamento em suco concentrado congelado ainda é pequeno. A conservação da fruta na forma de polpa congelada é uma alternativa viável para aumentar e diversificar o oferecimento da mesma, cujo preço não é compensador quando comercializada na maioria das vezes na forma natural.

De acordo com BRITO (2011), a comercialização de polpas de frutas no mercado interno e externo é interessante para a agroindústria brasileira, pois o Brasil cresceu nesta área e conquistou 


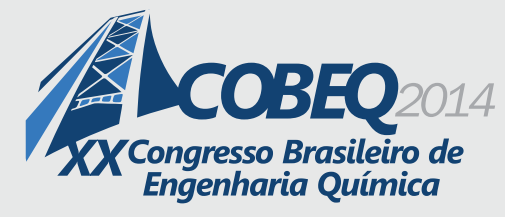

19 a 22 de outubro de 2014

Florianópolis/SC

a condição de maior exportador de polpa e sucos de frutas cítricas e tropicais, o que vem promovendo oportunidades de abertura de novos mercados para exportação de frutas e polpa de frutas congeladas do Brasil.

O congelamento é um dos métodos mais eficientes para a manutenção da qualidade de polpas de frutas (Fernandes et al., 2010). No entanto, fatores como velocidade de congelamento e meio de resfriamento produzem efeitos substanciais na qualidade do produto congelado (Colla e Prentice-Hernández, 2003). Segundo Matta et al., (2003) a velocidade de congelamento é um dos fatores mais importantes, visto que, os produtos biológicos apresentam teor de água elevado e o tamanho e a forma dos cristais de gelo estão relacionados as velocidades de congelamento.

No congelamento lento, a velocidade de congelamento vai decrescendo gradativamente (taxa de $1{ }^{\circ} \mathrm{C} / \mathrm{min}$.), havendo a formação de grandes cristais de gelo, exclusivamente no meio extracelular. Em altas taxas de congelamento (congelamento rápido), ocorre a formação de pequenos cristais de gelo nos espaços intercelulares e intracelulares, em grande quantidade, com mínimo deslocamento de água (Belchoir, 2012; Colla e Prentice-Hernández, 2003; Soares et al., 2012).

Durante o processo de congelamento da polpa de fruta, a temperatura recomendada é na faixa de $-23 \pm 5^{\circ} \mathrm{C}$, onde a maior parte do fluido contido é convertido em gelo. No entanto, o tempo necessário para abaixar a temperatura do produto para $-5^{\circ} \mathrm{C}$ não deve ultrapassar 8 horas. Essa temperatura deverá atingir cerca de $-18^{\circ} \mathrm{C}$ em um tempo máximo de 24 horas e deverá ser mantida durante todo o tempo de armazenamento e transporte até o momento do consumo (Moraes, 2006).

Considerando que não há um estudo científico da polpa de limão Tahiti e o seu uso em grande parte como especiaria, faz-se necessário a aplicação de técnica de congelamento como uma alternativa no mercado de polpa de fruta, possibilitando mais uma opção para o consumidor.

\section{MATERIAL E MÉTODOS}

Os experimentos foram realizados nos Laboratórios: Processamento e Armazenamento de Produtos Agrícolas da Unidade Acadêmica de Engenharia Agrícola da UFCG, Certificação de Biomateriais (CERTBIO) da Unidade Acadêmica de Engenharia de Materiais da UFCG e no Experimental de Térmica de Fluidos da Unidade Acadêmica de Engenharia Mecânica da UFCG.

Os frutos de limão Tahiti (Citrus latifolia Tanaka) foram adquiridos no comércio local (EMPASA) da cidade de Campina Grande-PB e transportados para o Laboratório de Processamento e Armazenamento de Produtos Agrícolas da Universidade Federal de Campina Grande (UFCG). Os limões foram selecionados de acordo com o grau de maturação, considerando a coloração e apresentação da superfície da casca, tamanho, firmeza e aparência. Os frutos excessivamente verdes, com injúrias teciduais e em estádio de maturação avançado foram descartados. Em seguida, foi feita a sanitização dos frutos com solução clorada a 50 ppm por 10 min, e enxague em água corrente para a retirada do excesso de cloro. Os limões foram descascados manualmente com facas de aço inoxidável e sua polpa foi extraída em uma despolpadeira horizontal de aço inoxidável, Modelo DF-200 (marca Laboremus), refinada em peneira com malha menor que $1,0 \mathrm{~mm}$, envasada em sacos plásticos de polietileno com capacidade de $100 \mathrm{~mL}$ e submetida ao processo de congelamento a $-20 \pm 2{ }^{\circ} \mathrm{C}$. 


\subsection{Cinética de Congelamento da Polpa de Limão Tahiti}

Para a realização da cinética de congelamento, as polpas foram levadas para o Laboratório Experimental de Térmica de Fluidos da unidade Acadêmica de Engenharia Mecânica da UFCG e congeladas em um freezer vertical doméstico da marca Cônsul com temperatura de $-20 \pm 2^{\circ} \mathrm{C}$. Durante o processo de congelamento, as temperaturas das amostras e do freezer foram monitoradas com termopares tipo K (cobre-constantan), sendo um posicionado dentro da amostra (centro geométrico) e o outro no ambiente do congelador. Os dados de temperatura foram registrados a cada minuto até que atingisse a temperatura de equilíbrio $\left(-20 \pm 2^{\circ} \mathrm{C}\right)$. $\mathrm{O}$ sistema de aquisição de dados NILabVIEW 8.2, mantinha conexão remota com o computador para o armazenamento dos dados. Os ensaios foram realizados em triplicata, e a partir dos dados de tempo-temperatura foram obtidas as curvas de congelamento.

\subsection{Análise das Microestruturas dos Cristais de Gelo}

A microestrutura dos cristais de gelo foi analisada, após congelamento das polpas, em um microscópio óptico (HIROX KH1300) acoplado a um SOFTWARE 2D Measure, disponível no Laboratório de Certificação de Biomateriais (CERTBIO) da Unidade Acadêmica de Engenharia de Materiais da UFCG. As imagens das estruturas cristalinas das polpas congeladas foram ampliadas para (100x e 200x) e fotomicrografadas para observação das estruturas dos cristais de gelo formadas no processo de congelamento a $-20 \pm 2{ }^{\circ} \mathrm{C}$.

\section{RESULTADOS E DISCUSSÃO}

\subsection{Cinética de Congelamento da Polpa de Limão}

Para a realização da cinética de congelamento, as polpas de limão foram resfriadas a partir da temperatura ambiente até $-20 \pm 2^{\circ} \mathrm{C}$. Efetuou-se o processo de congelamento em três ensaios, realizados em triplicata, para avaliação das curvas que melhor representassem a cinética de congelamento da polpa de limão, bem como, a determinação das temperaturas de início de congelamento.

A Figura 1 apresenta as curvas de congelamento construídas a partir dos dados de temperatura a $-20 \pm 2^{\circ} \mathrm{C}$, registrados durante os ensaios do processo de congelamento da polpa de limão Tahiti. Pode-se observar que as mesmas, foram similares à curva de congelamento obtida para a água pura. 


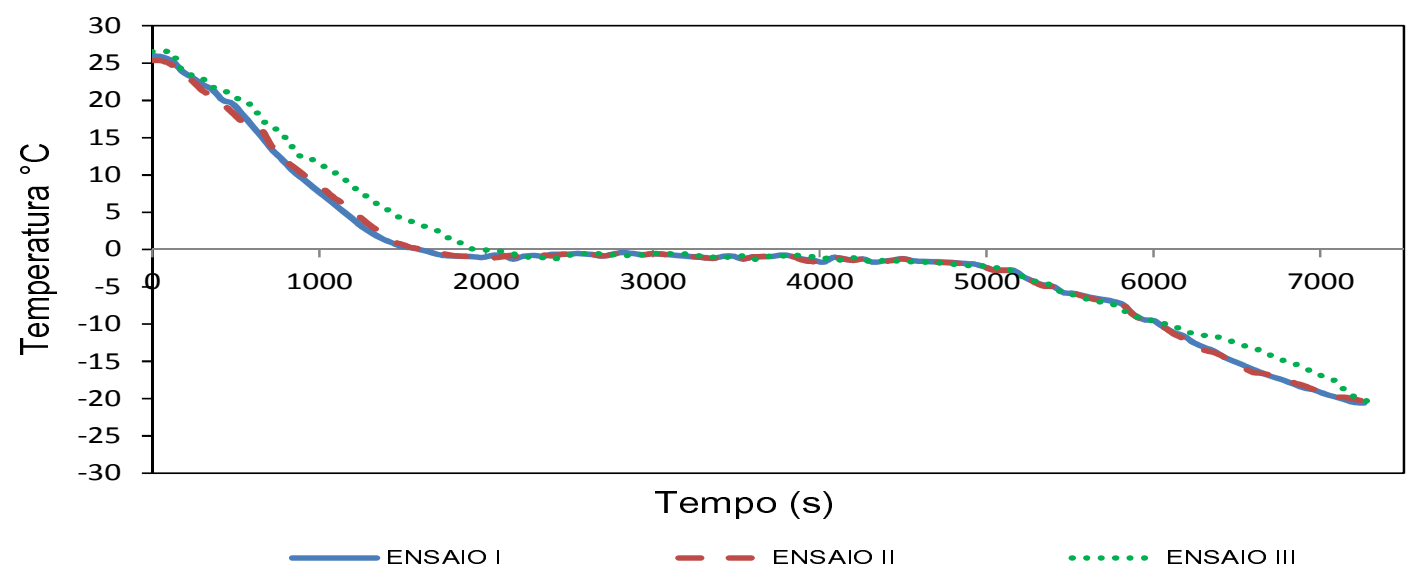

Figura 1 - Curvas dos ensaios de congelamento da polpa de limão.

Devido à baixa taxa de congelamento $\left(-20 \pm 2^{\circ} \mathrm{C}\right)$ foi possível verificar as três fases distintas da conversão da água contida no alimento em gelo, como: fase de resfriamento do produto, caracterizada por uma curva típica exponencial; fase de cristalização ou congelamento da água, representada por uma reta e a fase de pós congelamento, caracterizada por uma curva exponencial.

A partir das curvas de congelamento, foram obtidos os gráficos das taxas de resfriamento, apresentadas nas Figuras 2a, $2 \mathrm{~b}$ e 2c, para a determinação da temperatura de início de congelamento $\left(\mathbf{T}_{\mathbf{i c}}\right)$ de cada ensaio.

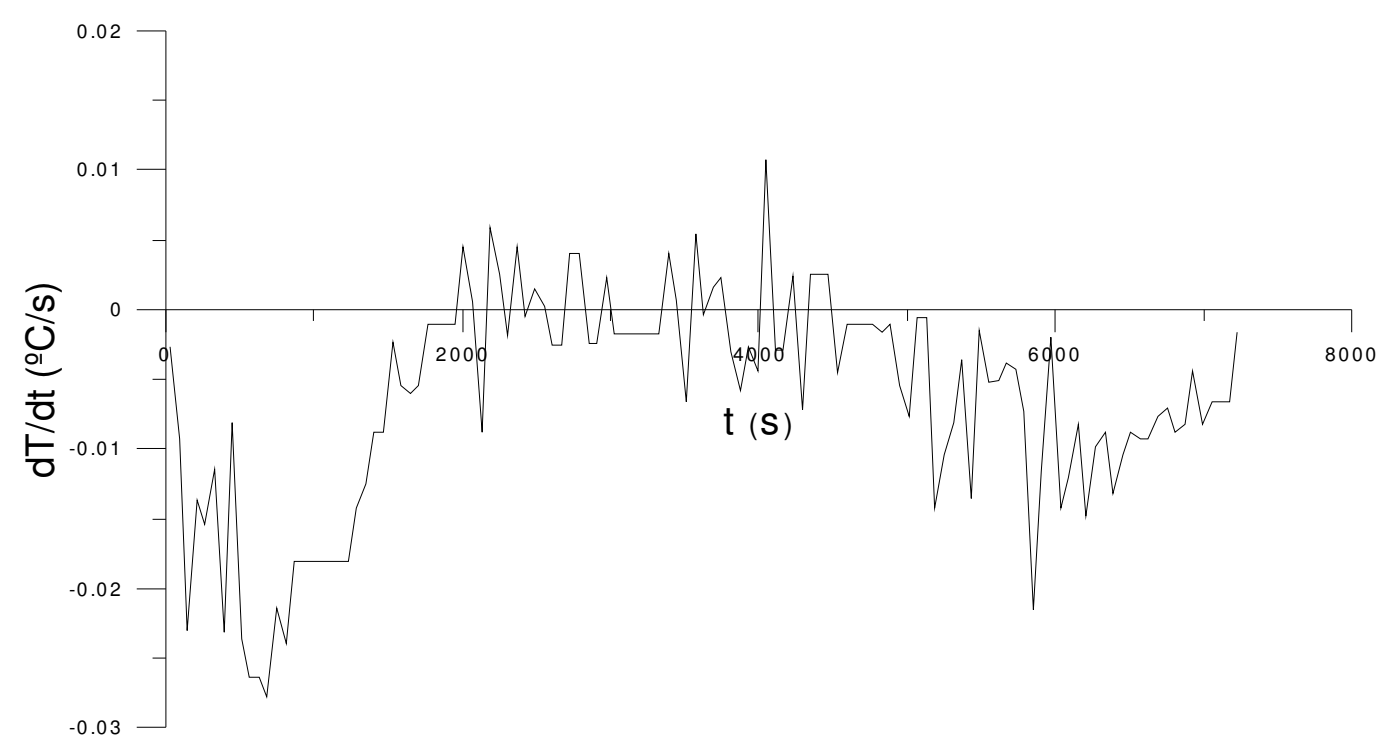

(2a) 


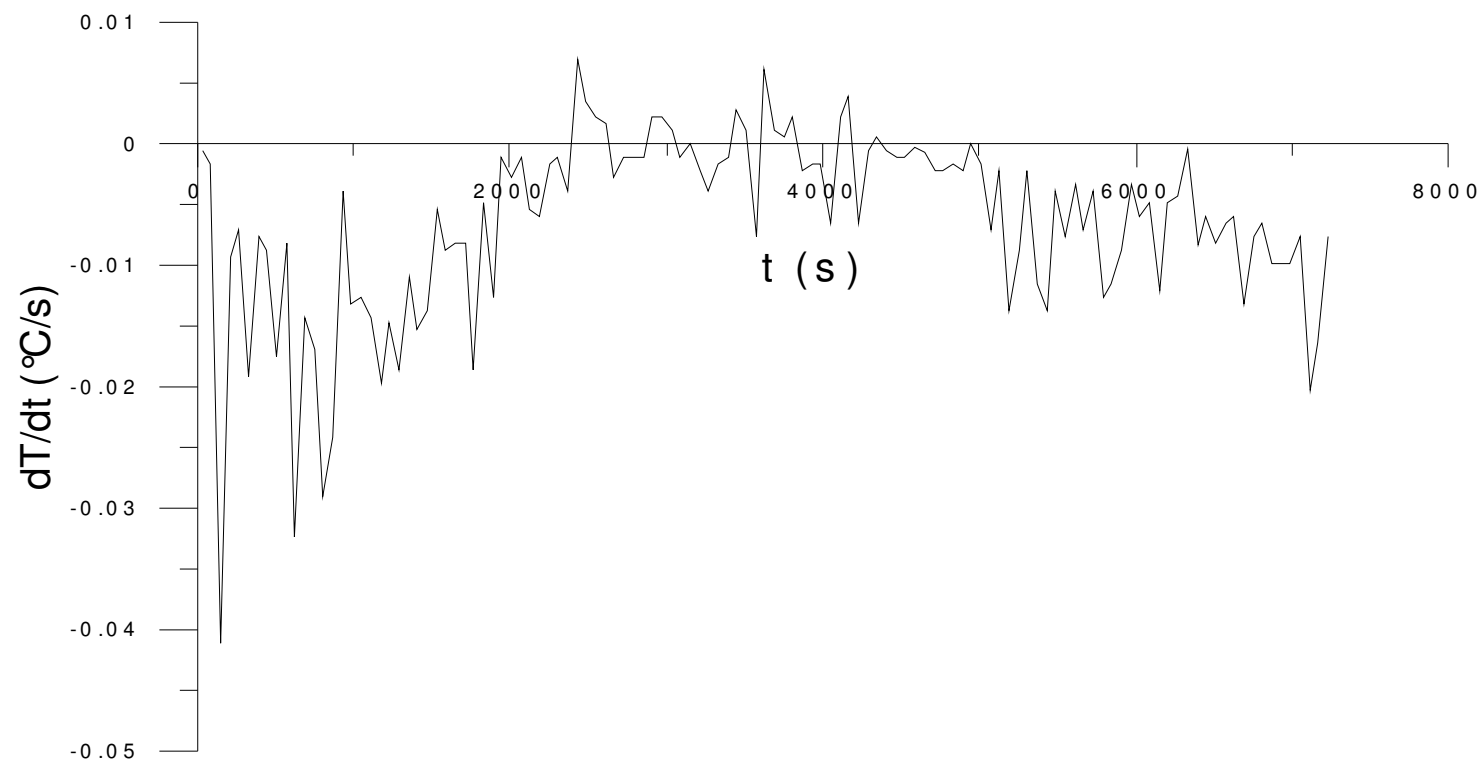

(2b)

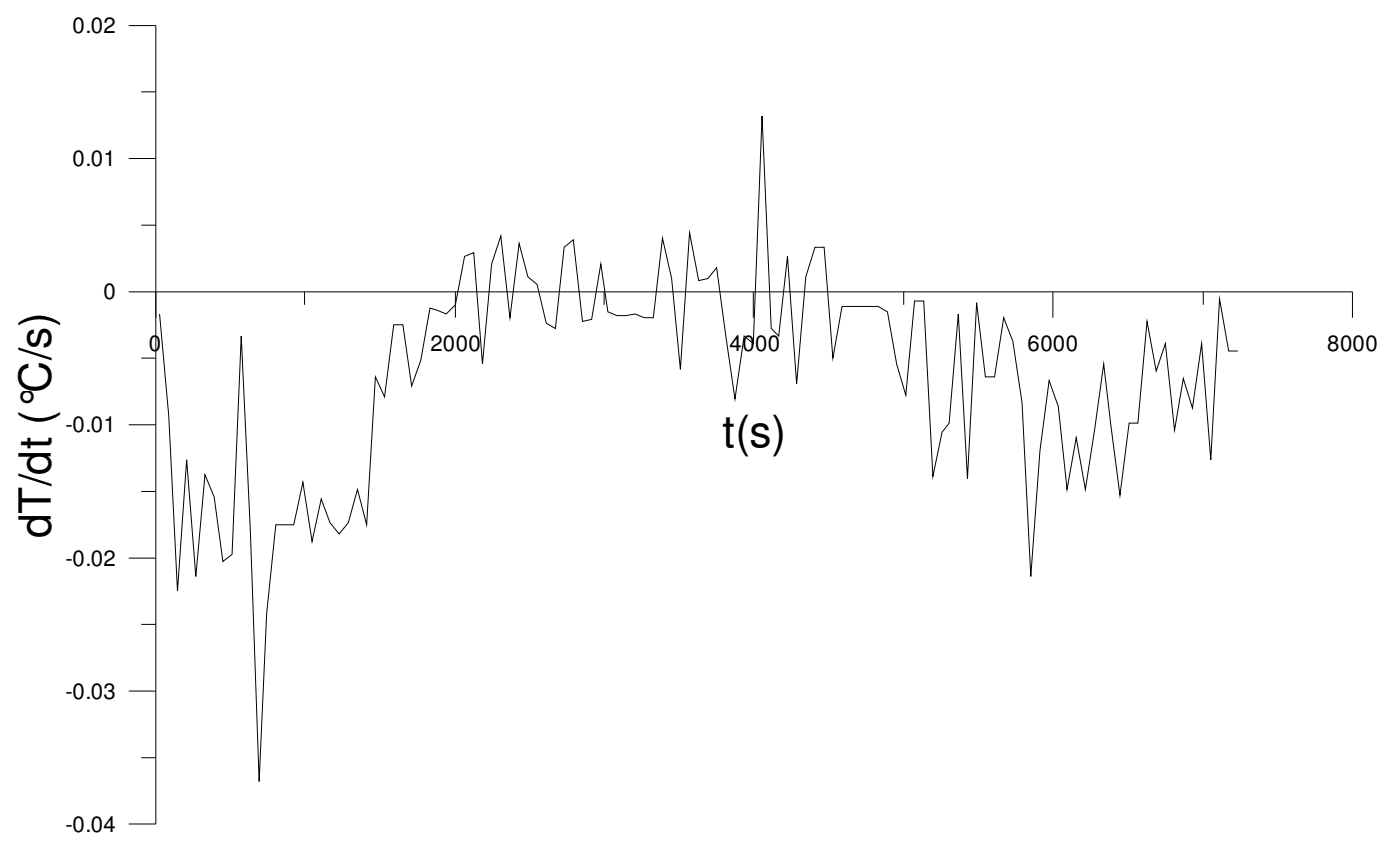

$(2 c)$

Figura 2 - Taxa de resfriamento da polpa de limão Tahiti durante o congelamento a $-20 \pm 2^{\circ} \mathrm{C}$. 


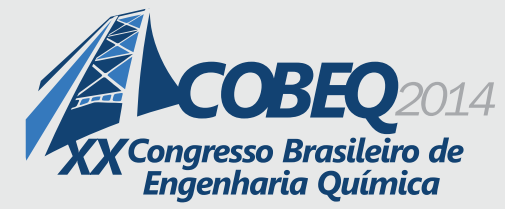

Os gráficos da taxa de resfriamento para cada ensaio evidenciaram que após o subresfriamento houve um aumento da temperatura em decorrência da formação dos primeiros cristais de gelo e liberação de calor latente. Neste intervalo, em que ocorreu o aumento da temperatura, obteve-se temperaturas de início de congelamento da polpa para os três ensaios de: $\mathrm{T}_{\mathrm{ic}}=-1,024^{\circ} \mathrm{C}(\mathrm{a}), \mathrm{T}_{\mathrm{ic}}=-1,045^{\circ} \mathrm{C}(\mathrm{b})$ e $\mathrm{T}_{\mathrm{ic}}=-1,032^{\circ} \mathrm{C}$ (c) para os tempos de 1999,2280 e 2000 segundos, respectivamente.

Fernandes et al. (2010) estudando o congelamento de polpa de morango em banho ultratermostático a $-20{ }^{\circ} \mathrm{C}$ verificaram uma temperatura de início de congelamento de $-0,7{ }^{\circ} \mathrm{C}$. Soares et al. (2012) utilizando um ultrafreezer para o congelamento da polpa de mangaba verificaram uma temperatura inicial de congelamento de aproximadamente $-1^{\circ} \mathrm{C}$. Já Pereira (2013) congelando polpa de acerola em câmara fria a $-25^{\circ} \mathrm{C}$, obteve uma temperatura de início de congelamento de $-1,1^{\circ} \mathrm{C}$. Araújo et al. (2000) no estudo do congelamento da polpa de acerola em um freezer com temperatura de $-22,6^{\circ} \mathrm{C}$ verificaram um período de 1.800 segundos para o resfriamento e 6.000 segundos para a cristalização e o pós-congelamento.

Avaliando os resultados obtidos para o tempo e a temperatura de início de congelamento da polpa de limão Tahiti, pode-se ressaltar que os valores experimentais determinados estão coerentes com os especificados na literatura.

\subsection{Análise Microestrutural}

As microestruturas dos cristais de gelo da água disponível da polpa de limão congelada a $20 \pm 2^{\circ} \mathrm{C}$, podem ser observadas na Figura $3 \mathrm{com}$ aumentos de 100 e 200x. Verifica-se que após o congelamento, os cristais de gelo apresentaram estruturas com ramificações semelhantes à de uma árvore, caracterizada como dendrítica, presente na grande maioria dos processos de solidificação.

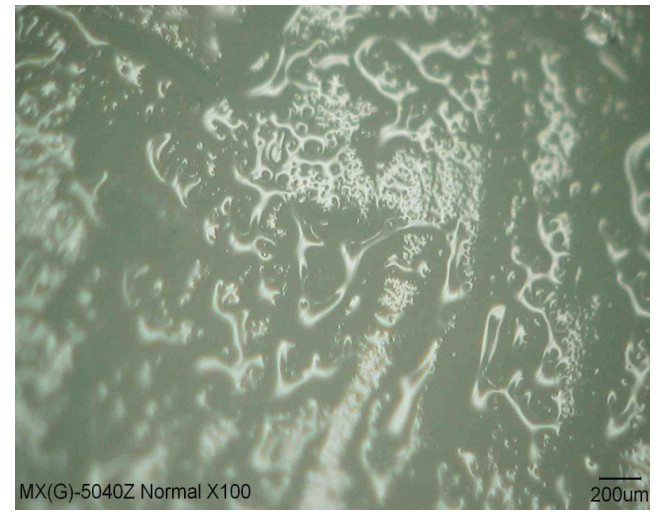

(a)

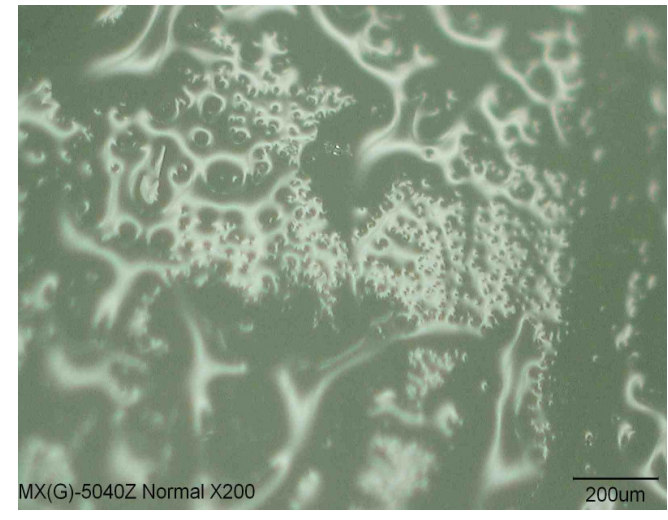

(b)

Figura 3 - Fotomicrografias da polpa de limão congelada a $-20 \pm 2{ }^{\circ} \mathrm{C}$ (a) aumento de 100x (b) aumento de 200x. 
As fotomicrografias mostram que os cristais de gelo configuraram uma rede de dendríticas que cresceram paralelamente e com espaços intercelulares presentes na estrutura celular. Esses espaços possibilitam o aumento no teor de água intracelular, que é favorável à formação de grandes cristais de gelo.

Segundo Rocha et al. (2003), o espaçamento dendrítico é inversamente proporcional à velocidade de resfriamento, pois quanto maior for essa velocidade, mais refinada será a microestrutura.

Logo, pode-se ressaltar que a velocidade de congelamento é reconhecida como um dos fatores responsáveis pela manutenção das características microestruturais do produto congelado, já que os fenômenos de nucleação e crescimento de estruturas dendríticas ocorrem ao longo da evolução do processo.

Além da dinâmica do processo de congelamento, os aspectos como heterogeneidade da composição química e concentrações dos componentes envolvidos também estão relacionados à intensidade do crescimento do cristal. Desta forma, é de grande importância o entendimento do desenvolvimento dendrítico durante o congelamento lento de polpas de frutas, já que as características das dendríticas e o controle de segregação que se forma nos espaços intercelulares irão influenciar fortemente nas propriedades do produto final.

\section{CONCLUSÕES}

As curvas de congelamento da polpa de limão foram similares as da água pura, apresentando temperaturas de início de congelamento de $-1,024^{\circ} \mathrm{C},-1,045^{\circ} \mathrm{C}$ e $-1,032^{\circ} \mathrm{C}$ correspondendo aos tempos de 1999, 2280 e 2000 segundos, respectivamente.

A microestrutura da polpa congelada de limão evidenciou a formação de grandes cristais de gelo nos espaços intercelulares, decorrente da baixa taxa de congelamento $(-20 \pm$ $\left.2^{\circ} \mathrm{C}\right)$.

\section{REFERÊNCIAS}

ARAÚJO, M. S. O.; BRAGA, M. E. D.; MATTA, M. E. R. M. Cinética de congelamento de polpa de acerola a baixas temperaturas. Revista Brasileira de Produtos Agroindustriais, Campina Grande, v.2, n.1, p.27-34, 2000.

BELCHIOR, N. C. Transferência de calor no congelamento de polpa de maracujá. 2012. 126p. Dissertação (Mestrado em Ciência dos Alimentos) - Universidade Federal de Lavras. Minas Gerais - MG.

BRITO, A. Relatório final de consultoria agroindústria de polpa de frutas. Recife, 2011. Disponível em: http://www.prorural.pe.gov.br/downloads/pesquisa.pdf Acesso em: 31 de março de 2013. 
COLLA, L. M.; PRENTICE-HERNANDEZ, C. Congelamento e Descongelamento: Sua Influência sobre os Alimentos. Vetor, Rio Grande, v.13, n. 53, p. 53-66, 2003.

FERNANDES, T. N.; RESENDE, J. V.; CRUVINEL, R. S. R.; RENO, M. J. Relação entre o comportamento reológico e a dinâmica do congelamento e descongelamento de polpa de morango adicionada de sacarose e pectina. Ciência e Tecnologia de Alimentos, Campinas, v.30, n.1, p.188-204, jan.-mar. 2010.

MATA, M. E. R. M. C.; BRAGA, M. E. D.; SILVA, M. da. Curvas de congelamento de frutos de cajá (Spondias lútea L.) a temperaturas semi-criogênicas. Revista Brasileira de Produtos Agroindustriais, Campina Grande, Especial, n.1, p.55-62, 2003.

MORAES, I. Dossiê técnico: Produção de polpa de fruta congelada e suco de frutas. Rede de Tecnologia do Rio de Janeiro, Outubro. 2006. Disponível em: http://www.respostatecnica.org.br/dossie-tecnico/downloadsDT/MTE3. Acesso em: 26 novembro. 2012.

PEREIRA, C. G. Propriedades termofísica e comportamento reológico de polpa de acerola em diferentes concentrações e temperaturas. 2013. 135p. Dissertação (Mestrado em Ciência de Alimentos) - Universidade Federal de Lavras - MG.

ROCHA, O. F. L.; SIQUEIRA, C. A.; GARCIA, A.; Heat Flow Parameters Affecting Dendrite Spacings During Unsteady State Solidification of $\mathrm{Sn}-\mathrm{Pb}$ and $\mathrm{Al}-\mathrm{Cu}$ Alloys. Metallurgical and Materials Transactions A, v.34, p. 995-1006, 2003.

SOARES, D. S. C.; SANTOS, J. T. S.; CAMPOS, A. F. P.; COSTA, F. S. C.; NUNES, T. P.; JÚNIOR, A. M. O. Avaliação do tempo de congelamento da mangaba (Hancornia Speciosa Gomes) em ultrafreezer através dos modelos matemáticos de Planck e Pham. Scien. Plena, v. 8, n.4. 2012. 